\title{
Interprétation en échographie mammaire : images hyperéchogènes
}

Ultrasound interpretation: hyperecchoic breast lesions

\author{
Martine Boisserie-Lacroix*, Marie-Pierre Depetiteville, Foucault \\ Chamming's
}

Unité de radiosénologie, département d'imagerie, département

d'anatomocytologie, institut Bergonié, centre de lutte contre le cancer, 229 cours de l'Argonne, 33076 Bordeaux cedex

* Auteur correspondant : Martine Boisserie-Lacroix

E-mail : m.boisserie-lacroix@ bordeaux.unicancer.fr 


\title{
Résumé
}

Les images mammaires hyperéchogènes sont connues pour avoir une valeur prédictive très élevée de bénignité. Néanmoins, en pratique quotidienne, on rencontre des lésions malignes entièrement ou en partie hyperéchogènes. Nous faisons le point ici sur les lésions mammaires bénignes et malignes, en insistant sur les signes qui peuvent faire évoquer une pathologie maligne sous-jacente.

\section{Mots-clés}

échographie mammaire - hyperéchogénicité - cancer du sein - histologie

\begin{abstract}
Hyperechoic lesions of the breast are benign in most cases. However some hyperechoic malignant lesions can be encountered in clinical practice. The purpose of this pictorial essay is to review hyperechoic benign and malignant breast lesions, focusing on features associated with malignancy.
\end{abstract}

\section{Keywords}

breast ultrasound - hyperechoic - malignancy - histology

\section{Introduction}

Si la grande majorité des lésions mammaires solides détectées par échographie sont hypoéchogènes, dans près de $6 \%$ elles apparaissent en partie ou totalité hyperéchogènes (1). D'après le lexique de Breat Imaging-Reporting and Data System (BI-RADS) de l'American College of Radiology (ACR), une lésion hyperéchogène se définit par son échogénicité supérieure à celle de la graisse sous-cutanée, ou encore égale à celle du cône conjonctivoglandulaire (2).

Longtemps interprétées comme bénignes (3), il s'avère que ces lésions hyperéchogènes peuvent être malignes, certes de façon rare mais à l'origine de pièges d'interprétation. Il convient de connaître les lésions bénignes et malignes pouvant se présenter sous forme de masses hyperéchogène, et de savoir repérer les signes de suspicion, d'autant qu'il peut s'agir d'images subtiles en échographie de second-look après l'IRM. 


\section{2. Évolution de la séméiologie}

Dès le début de l'utilisation de l'échographie dans la caractérisation des images, Stavros et al. dans une étude de référence analysant 750 nodules mammaires, ont attribué une valeur prédictive positive de $100 \%$ aux images «d'hyperéchogénicité intense » et homogène : aucun des 42 nodules présentant ces caractéristiques n'était malin (3). Cette étude de 1995 a été la pierre angulaire de l'interprétation prédictive des images et a été confortée par d'autres séries dans la décennie suivante.

Il a fallu attendre les années 2010 pour que des observations cliniques démentant cette certitude aboutissent à plusieurs publications : des cancers peuvent apparaître hyperéchogènes, dans moins de $1 \%$ de l'étude de Linda et al. (4). En pratique, cette situation semble plus fréquente, si on prend en compte des images modérément hyperéchogènes, des plages à prédominance hyperéchogène mais un peu hétérogènes, et les images de second-look subtiles ou atypiques mises en évidence grâce aux progrès des appareillages, autant d'images s'éloignant des caractéristiques décrites par Stavros et al.

\section{Quel substratum anatomique?}

L'hyperéchogénicité est en corrélation avec des composants ou des modifications tissulaires d'étiologies variées et parfois associées (4). Tous les mécanismes expliquant l'échogénicité ne sont cependant pas connus.

\subsection{Graisse}

À l'état liquide, de façon physiologique, des vacuoles graisseuses en suspension se trouvent dans le lait (galactocèle), dans les sécrétions dans des canaux. De la graisse à l'état liquide peut correspondre à la lyse de vacuoles graisseuses dans un kyste butyrique (cytostéatonécrose) post-traumatique ou post-thérapeutique (après une chirurgie et surtout une radiothérapie associée) (Figure 1).

A l'état solide, il peut s'agir d'une tumeur purement graisseuse, superficielle (n'envahissant pas le fascia profond) à type de lipome, ou de ses variantes (angiomyolipome). La graisse sous-cutanée est de la graisse «jaune » constituée d'adipocytes blancs ; elle est de distribution ubiquitaire (inter- et intramusculaire, médiastinale, péritonéale ; mais elle est alors hypoéchogène en échographie). 
La graisse brune, comportant des adipocytes bruns, impliquée dans la thermogenèse, est présente chez les mammifères hibernants et le nouveau-né jusqu'à ses 8 semaines de vie. Des tumeurs «brunes » exceptionnelles ont été décrites chez la femme adulte.

\subsection{OEdème}

Après un traumatisme, les lobules graisseux hypoéchogènes peuvent présenter une hyperéchogénicité diffuse.

\subsection{Fibrose du tissu conjonctif}

Il peut s'agit de plages de fibrose focale à l'intérieur du cône conjonctivo-glandulaire, ou à l'intérieur de certaines lésions bénignes bien limitées (hamartome, hyperplasie pseudoangiomateuse du stroma [pseudoangiomatous stromal hyperplasia, PASH]). L’hyperéchogénicité résulte des multiples interfaces entre tissu fibreux et adipeux (5).

La fibrose peut être partie intégrante de mastopathies complexes (radial scar). Elle peut aussi être réactionnelle, périphérique à un cancer : c'est la stroma réaction autour d'un carcinome canalaire in situ ou d'un cancer infiltrant.

\subsection{Hypercellularité tumorale}

L'hyperéchogénicité n'est pas due ici à la stroma réaction mais à la densité cellulaire élevée d'une tumeur bénigne (adénofibrome) ou maligne (carcinome invasif, lymphome, métastase).

\subsection{Composants particuliers : mucine, silicone}

\subsubsection{Quels signes rechercher et décrire?}

Il est nécessaire, pour l'interprétation de ces images, de connaître le contexte clinique (antécédent de traitement conservateur, de traumatisme) et les données de l'examen clinique ; d'analyser tous les caractères échographiques en échelle de gris comme usuellement, notamment le bord circonscrit ou indistinct voire spiculé, l'homogénéité ou l'hétérogénéité. La présence d'aires hypoéchogènes à l'intérieur d'une plage hyperéchogène augmente le risque de malignité (4) (Figure 2). Pour Nassar et al, les signes significativement suspects sont la forme irrégulière, l'orientation non parallèle, le bord indistinct (6). Pour ces auteurs, la taille de la lésion, les modifications postérieures du faisceau ultrasonore, l'existence d'antécédents personnels ou familiaux de cancer n'étaient pas corrélés au risque de cancer (6).

Le Doppler couleur peut être utilisé en complément, ainsi que l'élastographie, qui peut être déterminante, comme l'ont décrit par Chamming's et al. dans un article récent (7). 
Enfin, il convient de corrélera l'image avec la mammographie et si possible la tomosynthèse qui sera utile pour visualiser un contenu graisseux ; et avec l'IRM si cette dernière est indiquée.

\section{Orientation étiologique et conduite à tenir}

\subsection{Lésions bénignes}

\subsubsection{Galactocèle}

Son aspect dépend de la proportion des contenus graisseux et protéiniques en rétention dans un canal galactophore dilaté, un aspect hyperéchogène s'observant lors de contenu graisseux prédominant en corrélation avec une image claire mammographique. Il convient d'y penser en période d'allaitement ou de post-allaitement, mais aussi chez les patientes sous neuroleptiques (1). Ces images sont classées ACR 2. Le galactocèle se résorbe spontanément.

\subsubsection{Ectasie canalaire}

Le canal a un calibre dépassant $3 \mathrm{~mm}$, avec un aspect de comblement canalaire d'échogénicité variable selon la nature du contenu, échogène lorsque les sécrétions sont graisseuses (Figure 3). La mobilité des échos est un argument en faveur de sécrétions, de même l'absence de vascularisation. Ces images sont classées ACR 2. Quand l'aspect est ambigu, une ponction peut retirer un matériel jaune épais, pâteux.

\subsubsection{Cytostéatonécrose}

Quelle que soit sa cause, son aspect en imagerie est polymorphe, dépendant de la proportion de lyse graisseuse, hémorragie, infiltration histiocytaire, fibrose et calcifications. Une plage hyperéchogène peut être observée, dont l'analyse devra être rigoureuse afin de ne pas méconnaître une récidive dans un contexte de traitement conservateur (Figure 4). Des récidives précoces et hyperéchogènes de cancer triple négatif constituent des pièges diagnostiques. L'aspect de masse complexe à la fois liquide et solide est fréquente, avec des nodules échogènes donnant des aspects de pseudovégétations. Dans tous les cas, l'anamnèse et la corrélation avec la mammographie sont essentielles.

\subsubsection{Lipome}

Il est habituellement hypoéchogène de même échostructure que la graisse sous-cutanée, traversé par de fines travées. Moins souvent, le lipome est hyperéchogène, ce que Linda explique par une densité élevée d'adipocytes disposés de façon compacte ce qui crée de multiples interfaces (5). 
De petits lipomes péricentimétriques sont souvent visibles comme des inclusions dans les lobules graisseux superficiels, classés ACR 2 lorsqu'ils sont ovales, circonscrits et homogènes (Figure 5).

Plus volumineux, ces lipomes bénéficient de la même prise en charge que les masses graisseuses des parties molles ; cette stratégie a fait l'objet récemment d'une réactualisation (8). Compte tenu de la fréquence des lipomes superficiels, si les critères de bénignité sont réunis, et si la traduction mammographique est une image claire à la mammographie, le nouveau cut-off est établi à $10 \mathrm{~cm}$, taille à partir de laquelle une biopsie est recommandée (et non plus $5 \mathrm{~cm})$.

\subsubsection{Angio(myo)lipome}

De petits vaisseaux entrelacés sont associés à des adipocytes matures. Cette tumeur rare est habituellement de situation superficielle, ovale, circonscrite, homogène, iso- ou hyperéchogène (1). La microbiopsie peut être nécessaire quand tous les critères de bénignité ne sont pas réunis.

\subsubsection{Hibernome}

Quelques cas cliniques ont été rapportés dans la littérature, sous forme de lésion hyperéchogènes et homogènes, à type de masse bien circonscrite ou au contraire de plage à contour indistinct. Linda et al. ont ainsi rapporté un cas où l'indication d'une microbiopsie avec aiguille de calibre $14 \mathrm{G}$ avait été portée sur la forme irrégulière de la plage, en corrélation avec un rehaussement intense avec wash-out sur l'IRM (4) ; l'aspect en IRM de la graisse brune diffère en effet de celui de la graisse jaune, par son hypersignal T1 inférieur à celui de la graisse sous-cutanée et sa prise de contraste intense en rapport avec une vascularisation riche.

\subsubsection{Hématome}

Quelle qu'en soit l'origine, la traduction échographique dépend de l'âge du saignement, le caractère hyperéchogène s'observant à un stade chronique (Figure 6). La corrélation clinique est essentielle, d'autant que la traduction mammographique peut être ambiguë.

\subsubsection{Hamartome}

L'apparence dépend de la quantité de graisse. Typiquement il s'agit d'une masse encapsulée qui alterne des plages hypo- et hyperéchogènes (Figure 7). Mais dans 12 à $48 \%$ des cas, la tumeur peut être entièrement hyperéchogène (9). Le bord circonscrit, l'existence d'une 
capsule fine et un aspect mou en élastographie sont rassurants ; une biopsie s'envisagera si la masse est récente, palpable, et d'aspect mammographique non typique.

\subsubsection{Myofibroblastome}

Sur le plan histologique, on observe des cellules fusiformes en amas séparées par des bandes plus ou moins épaisses de tissu collagène. En échographie cette tumeur rare apparaît à contours circoncrits mais parfois indistincts, isoéchogène ou hyperéchogène ; dans ce dernier cas, une atténuation postérieure peut s'observer (10) (Figure 8). L'exérèse complète de la lésion par macrobiopsie permet une simple surveillance ; dans le cas contraire, une tumorectomie est nécessaire en raison des difficultés de diagnostic différentiel avec un carcinome lobulaire infiltrant.

\subsubsection{Adénofibrome}

Certaines de ces tumeurs bénignes peuvent être plus échogènes que la graisse, voire hyperéchogènes (Figure 9). C'est le cas chez la jeune fille ou la jeune femme. Il s'agit alors de tumeurs très cellulaires.

\subsubsection{Hyperplasie pseudoangiomateuse du stroma (PASH)}

Cette lésion mésenchymateuse bénigne est formée de cellules fibroblastiques fusiformes dans un abondant stroma fibreux, organisées autour de collections de polysaccharides optiquement vides, ce qui donne un faux aspect de réseau vasculaire. Elle est fréquemment infraradiologique ; la forme nodulaire est plus rare, unique ou multiple, unie ou bilatérale : masses de morphologie bénigne, sans microcalcification, hypo- ou hyperéchogènes, homogènes ou comportant quelques zones kystiques (Figure 10).

Certaines PASH sont probablement étiquetées adénofibromes. L'évolution spontanée des PASH est variable : stables dans plus de $80 \%$ des cas (suivi allant de 6 à 60 mois) ou augmentation progressive de taille (11). Des modifications morphologiques au cours du temps (contours lobulés, zones liquidiennes) font indiquer une microbiopsie avec aiguille de calibre 14G. On ne dispose pas de recommandation sur la surveillance spécifique.

\subsubsection{Fibrose focale}

Il s'agit d'une entité clinico-anatomopathologique définie par une prolifération fibreuse du stroma oblitérant les structures lobulaires et canalaires, pouvant se traduire cliniquement par une masse palpable chez une femme jeune. Sa traduction échographique est variée : masse circonscrite ou à bord indistinct, hypo- ou hyperéchogène : dans ce dernier cas, on invoque la 
présence de bandes épaisses fibreuses sans interposition de graisse, créant de multiples interfaces irrégulières d'impédance acoustique variée (3). L'image sera classée ACR 3 ou ACR 4a quand tous les critères de bénignité ne sont pas réunis.

\subsubsection{Mastopathies bénignes}

L'adénose peut se traduire par une plage hyperéchogène, de caractère plutôt bénin quand il s'agit d'adénose simple ou avec signes de suspicion pour l'adénose sclérosante où la fibrose désorganise l'architecture lobulaire. La conduite à tenir dépendra de la classification ACR échographique et mammographique.

La cicatrice radiaire est définie par un centre scléroélastosique vers lequel convergent des unités terminales ductulolobulaires ; on parle de lésion sclérosante complexe quand la fibrose est importante. La traduction échographique est inconstante, parfois sous forme d'une plage échogène ; l'existence d'une distorsion architecturale et de petits kystes peut orienter le diagnostic. Des prélèvements sont nécessaires, par macrobiopsies pour ne pas méconnaître une sous-estimation (lésions atypiques, carcinome canalaire in situ voire infiltrant).

\subsubsection{Hémangiome}

Cette tumeur est habituellement superficielle, échographiquement ovale ou lobulée, circonscrite ; elle présente plus rarement des microlobulations et un bord indistinct. Jusqu'à $33 \%$ de ces tumeurs sont hyperéchogènes, avec possible atténuation postérieure (12). Le Doppler couleur ne permet pas forcément de repérer des vaisseaux ; quand ceux-ci sont visibles, ils peuvent être périphériques ou intralésionnels.

\subsection{Lésions malignes}

Lorsqu'une lésion hyperéchogène correspond à un cancer canalaire invasif, sa forme est irrégulière, son orientation non-parallèle, et elle comporte habituellement un petit «nidus » central hypoéchogène (4). Ce petit centre hypoéchogène doit être repéré car il rend l'image suspecte ; en outre, c'est l'ensemble de la lésion qui doit être mesuré pour ne pas sous-estimer sa taille (Figure 11). Un bord spiculé, une distorsion architecturale des tissus avoisinants augmente la valeur prédictive de malignité. Certains phénotypes sont plus souvent en cause, ainsi les cancers luminaux qui s'accompagnent d'une forte réaction stromale.

Quand il s'agit d'une masse circonscrite à prédominance hyperéchogène, l'hétérogénéité doit être un signe de suspicion (Figure 12).

\subsubsection{Carcinomes lobulaires invasifs}


Les carcinomes lobulaires invasifs sont plus souvent associés à un aspect hyperéchogène : 9,9 fois plus souvent que les cancers canalaires invasifs en prenant en compte les lésions au moins partiellement hyperéchogènes (13) (Figure 13). Dans la série de Jones et al., $5 \%$ des 509 carcinomes lobulaires invasifs apparaissaient hyperéchogènes (14). L'infiltration périductale serait à l'origine de multiples réflexions des ultrasons autour du petit centre tumoral jusqu'à devenir le signe prédominant (14).

\subsubsection{Carcinomes mucineux}

Le carcinome mucineux dans sa forme pure apparaît isoéchogène, le contenu mucineux pouvant mimer une tumeur bénigne, voire légèrement hyperéchogène (15) (Figure 14).

À noter que l'hyperéchogénicité n'a pas d'implication en termes de pronostic (6).

Les récidives de carcinome infiltrant peuvent avoir un aspect hyperéchogène, à ne pas confondre avec un placard de fibrose postradique. L'irrégularité des bords, l'hétérogénéité même discrète, l'apparition de l'image sont autant de signes de suspicion (Figure 15).

\subsubsection{Angiosarcomes, métastases et lymphomes primitifs ou secondaires}

Les angiosarcomes, les métastases et les lymphomes primitifs ou secondaires sont décrits comme hypoéchogènes, mais ils peuvent parfois être d'échostructure mixte, voire de façon exceptionnelle entièrement hyperéchogènes $(16,17)$. Ils sont habituellement vascularisés.

\section{Conclusion}

L'analyse échographique des plages ou des masses hyperéchogènes doit être la même que celles des images hypoéchogènes, l'existence d'un signe suspect concernant la forme ou le bord devant faire classer ACR 4 afin de ne pas méconnaître les rares cancers hyperéchogènes.

\section{Points à retenir}

- L'analyse d'une plage hyperéchogène doit intégrer tous les signes habituels, notamment le bord.

- Un piège est le «nidus » hypoéchogène entouré d'une plage hyperéchogène dans un cancer canalaire invasif.

- L L'élastographie doit être pratiquée en complément. 


\section{Aucun conflit d'intérêts}

\section{Bibliographie}

1. Journo G, Bataillon G, Benchimol R, Bekhouche A, Dratwa C, Sebbag-Sfez D et al. Hyperechoic breast images: all that glitters is not gold! Insights Imaging 2018; 9: 199-209.

2. American College of Radiology. Illustrated breast imaging reporting and date system (BIRADS), 3rd edn. Reston: American College of Radiology, 2013.

3. Stavros AT, Thickman D, Rapp CI, Denis MA, Parker SH, Sisney GA. Solid breast nodules: use of sonography to distinguish between benign and malignant lesions. Radiology 1995; 196: 123-124.

4. Linda A, Zuiani C, Lorenzon M, Furlan A, Girometti R, Londero V et al. Hyperechoic lesions of the breast: not always benign. AJR 2011; 196: 1219-1224.

5. Linda A, Zuiani C, Lorenzon M, Furlan A, Londero V, Machin P et al. The wide spectrum of hyperechoic lesions of the breast. Clin Radiol 2011; 66: 559-565.

6. Nassar L, Ghada I, Farah Z, El Zein Y, Berjawi G. Predictors of malignancy in hyperechoic breast lesions. J Ultrasound Med 2016; 35: 783-790.

7. Chamming's F, Rousseau C; Depetiteville M-P, Boisserie-Lacroix M. Elastographie shear wave en sénologie : faux négatifs, faux positifs, comment optimiser l'examen ? Imagerie de la Femme $2018 ; 28: 226-236$.

8. Fouque O, Kind M, Boulet B, Brisse H, Kemel S, Genah I et al. Stratégie diagnostique devant une tumeur graisseuse des tissus mous de l'adulte. J Imag Diagn Interv 2018 ; 1 : 265283.

9. Chao TC, Chao HH, Chean MF. Sonographic features of breast hamartomas. J Ultrasound Med 2007; 26: 447-52.

10. Porter GJ, Evans AJ, Lee AH, Hamilton LJ, James JJ. Unusual benign breast lesions. Clin Radiol 2006; 61: 562-569.

11. Mercado CL, Naidrich SA, Hamele-Bena D, Fineberg SA, Buchbinder SS. Pseudoangiomatous stromal hyperplasia of the breast: sonographic features with histopathologic correlation. Breast 2004; 10: 427-32. 
12. Glazebrook KN, Morton MJ, Reynolds C. Vascular tumors of the breast: mammographic, sonographic, and MRI appearances. AJR 2005; 184: 331-338.

13. Cawson JN, Law EM, Kavanagh AM. Invasive lobular carcinoma: sonographic features of cancers detected in a BreastScreen Program. Australas Radiol 2001; 45: 25-30.

14. Jones KN, Magut M, Henrichsen TL, Boughey JC, Reynolds C, Glazebrook KN. Pure lobular carcinoma of the breast presenting as hyperechoic mass: incidence and imaging characteristics. AJR 2013; 201: W765-9.

15. Memis A, Ozdemir N, Parildar M, Ustun EE, Erhan Y. Mucinous (colloid) breast cancer: mammographic and US features with histologic correlation. Eur J Radiol 2000; 35: 39-43. 16. Tiang S, Metcalf C, Dissanayake D, Wylie E. Malignant hyperechoic breast lesions at ultrasound: A pictorial essay. J Med Imaging Radiat Oncol 2016; 60: 506-513.

17. Wiseman C, Liao K. Primary lymphoma of the breast. Cancer 1972; 29: 1705-12. 


\section{Légendes des figures}

Figure 1. Traitement conservateur du sein 6 mois avant, palpation d'un nodule péricentimétrique sous-cicatriciel. Echographie mammaire montrant une masse à prédominance hyperéchogène avec image de niveau : kyste butyrique, lésion ACR 2.

Figures 2. A : Echographie mammaire en échelle de gris : masse centimétrique hyperéchogène avec un centre hypoéchogène ; $\mathrm{b}$ : Elasticité intermédiaire en élastographie. Lésion ACR 4. Le résultat de la microbiopsie sous échographie a permis de diagnostiquer un cancer canalaire invasif grade 2 de phénotype luminal A.

Figures 3. Échographie mammaire : écoulement multipore laiteux. a : canal ectasié pseudokystique ; b : autre canal un peu dilaté, de contenu mobile en échoscopie. Lésion ACR 2.

Figures 4. Écoulement unipore clair droit à bilan conventionnel normal. L'IRM ne montre pas de prise de contraste à droite. a : IRM coupe pondérée T2 montrant une masse de $8 \mathrm{~mm}$ quadrant supérérieur interne gauche, hypointense $; b$ : IRM après injection de produit de contraste et soustraction montrant un rehaussement précoce et persistant ; c, échographie montrant une plage hyperéchogène à bord indistinct, un peu hétérogène, en possible corrélation ; d : élastographie shear wave, élasticité intermédiaire, lésion ACR 4a ; e : microbiopsie sous échographie avec pose de clip. L'histologie a identifié une cytostéatonécrose. Il n'y avait pas de cause mise en évidence à l'interrogatoire. Une IRM de contrôle de la pose du clip a permis de vérifier la concordance des images. Par la suite, la prise de contraste et la plage échogène ont disparu.

Figure 5. Masse infraclinique, infracentimétrique, de découverte fortuite, sans traduction mammographique, ovale, circonscrite, hyperéchogène, superficielle, avasculaire, souple : lipome, lésion ACR 2.

Figure 6. Accident de la voie publique avec choc de la ceinture de sécurité et hématome mammaire. Apparition d'une hyperdensité focale mammographique 1 an après.

L'échographie mammaire montre une plage hyperéchogène de $4 \mathrm{~cm}$, avec un centre liquidien centimétrique. Le résultat de la microbiopsie a montré des remaniements granulomateux chroniques. La patiente a fait l'objet d'une surveillance simple ; les images ont disparu au bout de 2 ans. 
Figure 7. Nodule mammaire palpable mobile. Échographie montrant une masse d'échostructure mixte à prédominance échogène, encapsulée, souple : hamartome, lésion ACR 2.

Figure 8. Mammographie de dépistage. a : masse comportant une clarté graisseuse ; b : échographie avec élastographie montrant une masse échogène de $15 \mathrm{~mm}$ avec atténuation postérieure, la composante échogène étant souple. La microbiopsie sous échographie a permis de diagnostiquer un myofibroblastome. Une macrobiopsie complémentaire a confirmé le diagnostic.

Figure 9. Mammographie normale. Découverte d'une masse échographique centimétrique. a : masse hyperéchogène, présentant une microlobulation antérieure ; $\mathrm{b}$ : discrète hétérogénicité. La masse était souple en élastographie. La microbiopsie sous échographie a mis en évidence un foyer d'adénose sclérosante. Une macrobiopsie complémentaire a permis de diagnostiquer un adénofibrome. Une surveillance habituelle a été poursuivie.

Figure 10. Masse mammaire palpable de $8 \mathrm{~cm}$. Echographie : masse ovale circonscrite, avec plages hyperéchogènes, avasculaire, souple. Le résultat de la microbiopsie a mis évidence une hyperplasie pseudoangiomateuse du stroma (pseudoangiomatous stromal hyperplasia, PASH). Une exérèse chirurgicale a été réalisée en raison de la taille.

Figure 11. Masse mammaire palpable droite. a : mammographie montrant une masse irrégulière difficile à délimiter avec une partie claire ; b : échographie montrant une masse irrégulière à prédominance hyperéchogène $; \mathrm{c}$ : contenu hétérogène. Lésion ACR $4 \mathrm{c}$. Le résultat de la microbiopsie a permis de diagnostiquer un cancer canalaire invasif HER2 enrichi.

Figure 12. Masses mammaires palpables. a : masse microlobulée hyperéchogène, hétérogène ; $b$ : seconde masse de même échostructure. Lésion ACR 4c. Le résultat de la microbiopsies a permis de diagnostiquer un cancer canalaire invasif de phénotype triple négatif.

Figure 13. Hyperdensité mammographique sans traduction échographique, IRM pratiquée hors centre mettant en évidence une masse suspecte en corrélation. a : Echographie de secondlook montrant une plage hyperéchogène un peu hétérogène ; $b$ : IRM montrant une masse très dure, lésion ACR 4c. Le résultat de la microbiopsie a permis de diagnostiquer un carcinome lobulaire invasif. 
Figure 14. a : échographie mammaire montrant une masse légèrement plus échogène que les lobules adipeux, un peu hétérogène ; $b$ : masse souple en élastographie. Lésion ACR 4b. Le résultat de la microbiopsie a permis de diagnostiquer un carcinome mucineux.

Figure 15. Antécédent de mastectomie pour cancer canalaire invasif. Palpation par la patiente d'un cordon induré du creux axillaire, décrit comme apparu. Echographie en échelle de gris montrant une plage échogène en corrélation avec l'examen clinique, un peu hétérogène : lésion ACR 4a. Le résultat de la microbiopsie a permis de diagnostiquer un cancer canalaire invasif. 


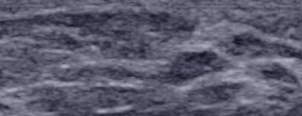

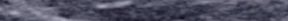

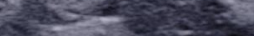

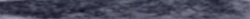

\section{+Dist}

tDist

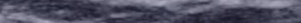

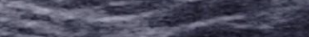

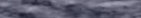

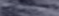

3

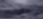

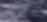

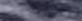

$\cos ^{2}+\sin ^{2}$

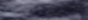

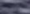

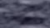




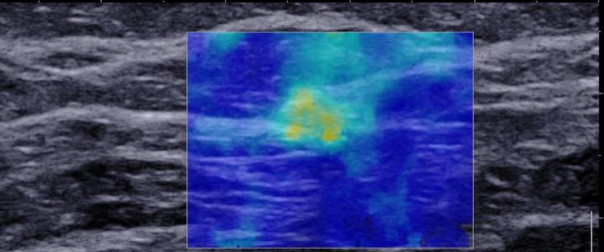

matios

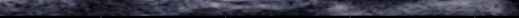

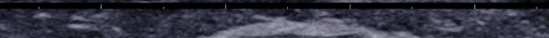

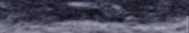

$\cos =1$

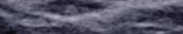

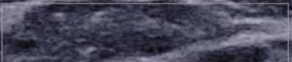

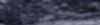

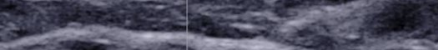

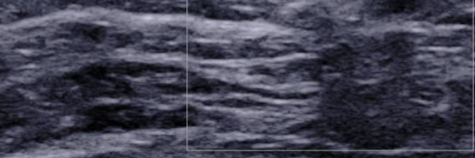

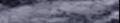

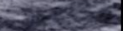
4

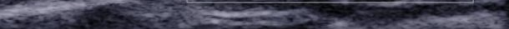




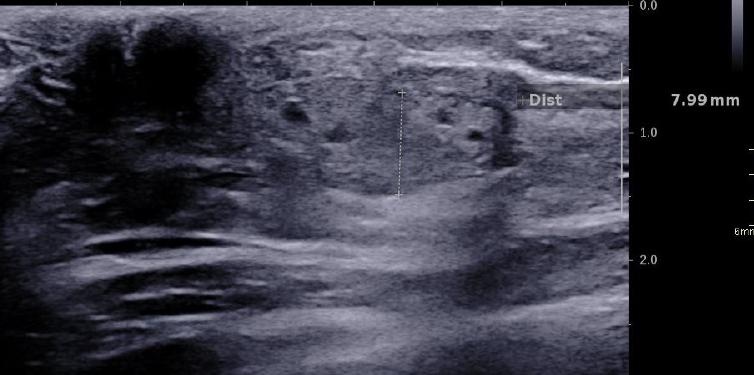





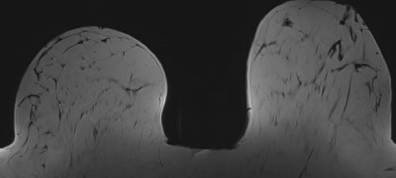




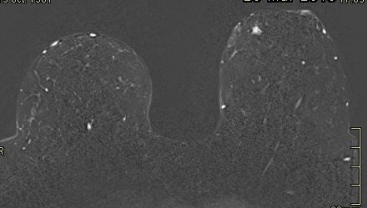

90in

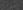



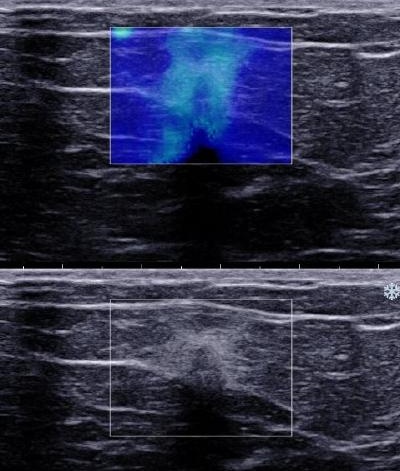



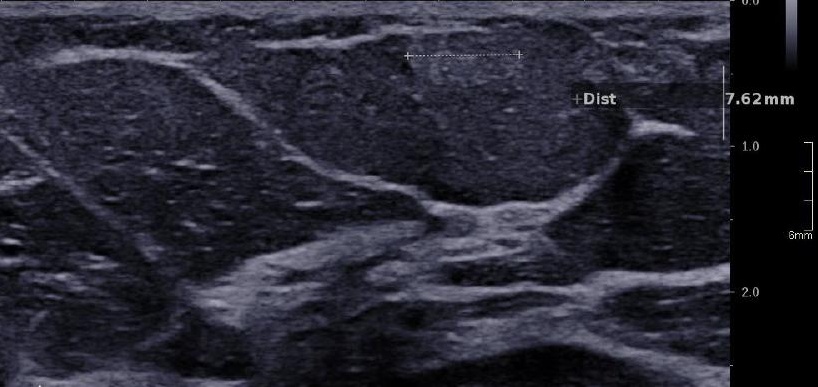




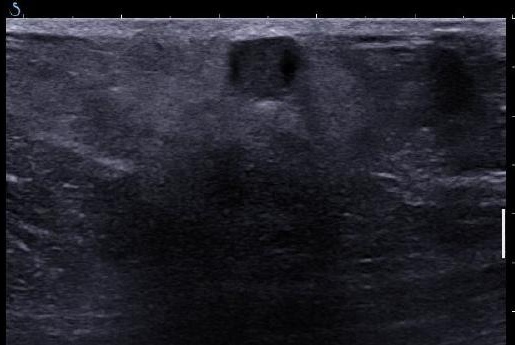


J

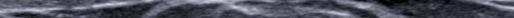

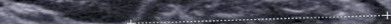

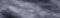

\section{$+$}
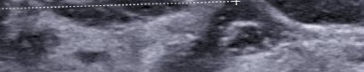

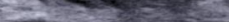

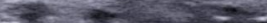

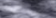

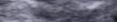

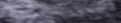

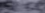

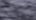

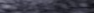

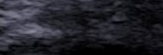

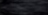

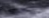

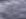

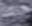

$-1$
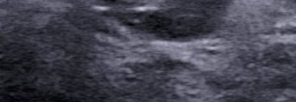
[E RMLO] 


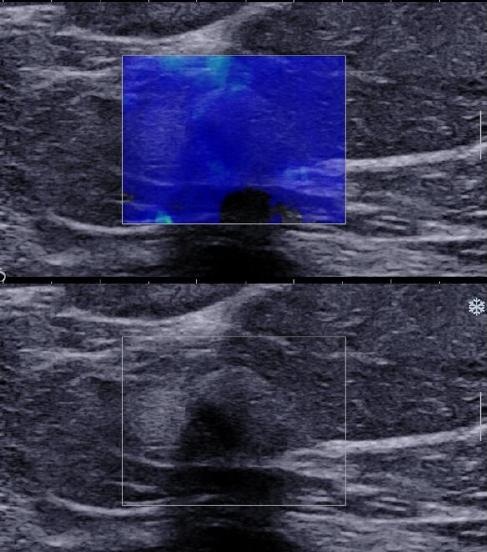




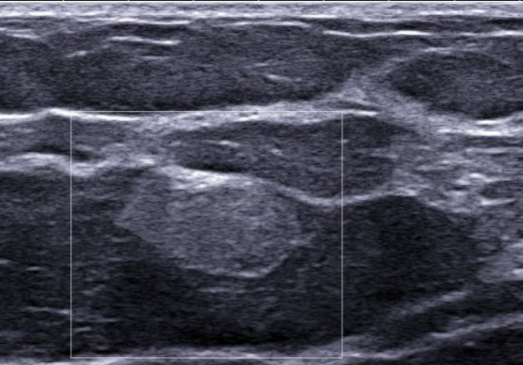




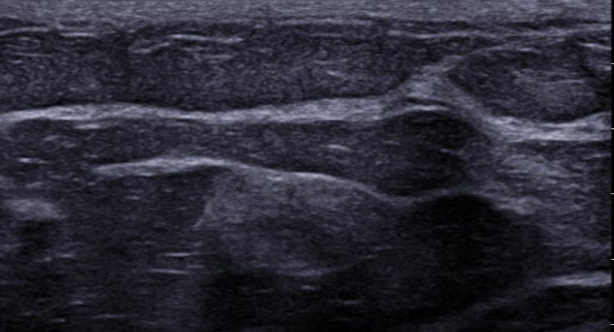




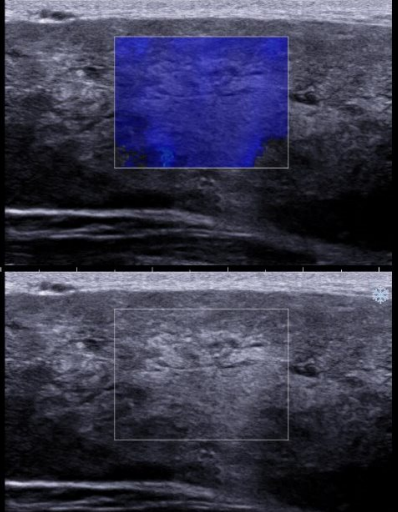


$\mathrm{RCC}^{7}$ 


\section{+Dist}

\section{$21.85 \mathrm{~mm}$}

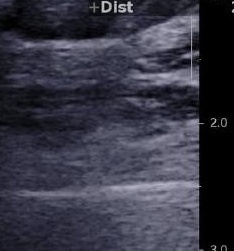




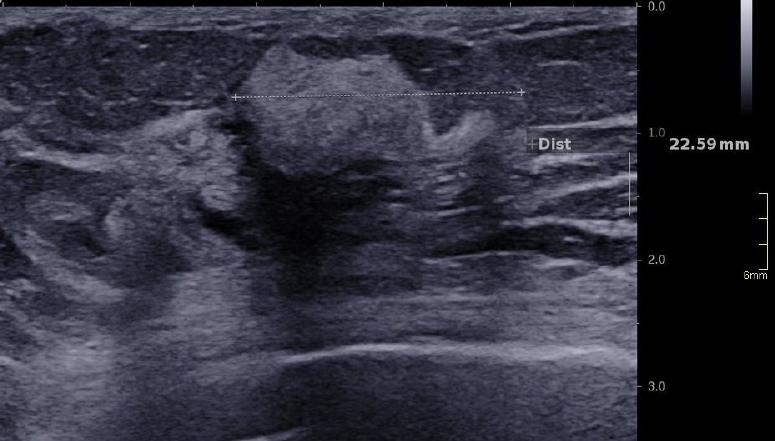


SC/SR 2

G $48 \%$

$\mathrm{Cl} 50 \mathrm{~Hz}$

Z $100 \%$

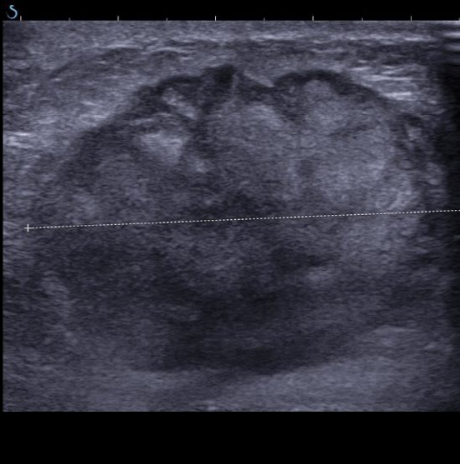


$638 \%$

Cl $50 \mathrm{~Hz}$

\section{Z $100 \%$}

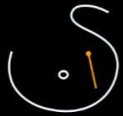

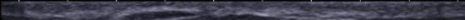

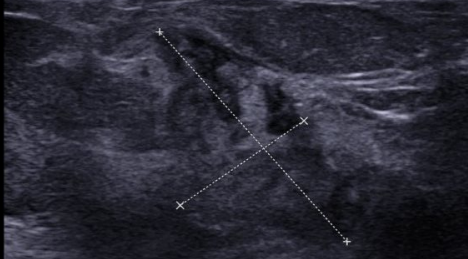







\section{B}

Geen/Moy
M 5/65 dB/Moy

$\mathrm{T} 1480 \mathrm{~m} / \mathrm{s}$

SCISR 2

G $25 \%$

Cl $59 \mathrm{~Hz}$

Z $120 \%$
5

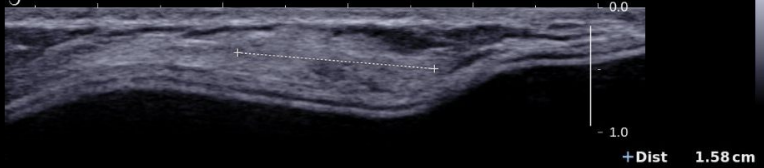

$-2.0$

$-3.0$

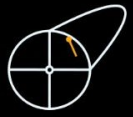

